\title{
Mathematical formulation of energy minimization - based inverse optimization
}

\section{Ivaylo B. Mihaylov *}

Department of Radiation Oncology, University of Miami, Miami, FL, USA

Edited by:

Thomas FitzGerald, University of Massachusetts, USA

\section{Reviewed by:}

Dalong Pang, Georgetown University Hospital, USA

Shirin Sioshansi, UMass Memorial

Medical Center, USA

\section{*Correspondence:}

Ivaylo B. Mihaylov, Department of Radiation Oncology, University of Miami, 1475 NW 12th Avenue, Suite 1500, Miami, FL 33136, USA

e-mail: i.mihaylov@med.miami.edu
Purpose: To introduce the concept of energy minimization-based inverse optimization for external beam radiotherapy.

Materials and Methods: Mathematical formulation of energy minimization-based inverse optimization is presented. This mathematical representation is compared to the most commonly used dose-volume based formulation used in inverse optimization. A simple example on digitally created phantom is demonstrated. The phantom consists of three sections: a target surrounded by high and low density regions. The target is irradiated with two beams passing through those regions. Inverse optimization with dose-volume and energy minimization-based objective functions is performed. The dosimetric properties of the two optimization results are evaluated.

Results: Dose-volume histograms for all the volumes of interest used for dose optimization are compared. Energy-based optimization results in higher maximum dose to the volumes that are used as dose-limiting structures. However, the average and the integral doses delivered for the volumes outside of the target are larger with dose-volume optimization.

Conclusion: Mathematical formulation of energy minimization-based inverse optimization is derived. The optimization applied on the digital phantom shows that energy minimizationbased approach tends to deliver somewhat higher maximum doses compared to standard of care, realized with dose-volume based optimization. At the same time, however, the energy minimization-based optimization reduces much more significantly the average and the integral doses.

Keywords: dose, volume, mass, energy, integral dose, inverse optimization

\section{INTRODUCTION}

The basic principle of external beam radiotherapy involves irradiation from a number of different directions (cross-firing) with beams of uniform or non-uniform energy fluences (intensities). The aim of this arrangement is to deliver a high dose to the target volume, while delivering as low doses as possible to the surrounding normal tissues. Radiotherapy dose calculations are based upon the following framework. CT derived attenuation coefficients (or Hounsfield Units) are mapped to electron density through a calibration procedure. The electron density (which scales with physics density of the material) governs the number of photon Compton interactions. The electrons, set in motion due to those Compton interactions, lead to ionizations, which affect the underlying biological response in the living cells and in particular lead to cell kill.

By its very definition dose is the radiation energy imparted per unit mass of material $(\mathrm{Gy}=\mathrm{J} / \mathrm{kg})$. The volume integral of the deposited dose therefore has units of energy and is also known as "integral dose." Alternatively in the discrete case applicable in radiotherapy, if dose is multiplied by mass on a dose voxel-by-voxel basis, and a summation over all dose voxels within a volume of interest (VOI) is performed, then the total energy imparted to that VOI would be obtained. It is often mentioned in the literature that the large number of beamlets and monitor units used in intensity modulated radiotherapy (IMRT) leads to an increase in integral dose compared to conformal radiotherapy (3DCRT) $(1,2)$. Furthermore, it is also commonly assumed that higher-energy photon beams substantially reduce the integral dose to normal tissue (3). However, an alternative hypothesis suggests that the total energy deposited in a patient during irradiation is relatively independent of treatment planning parameters, with some reduction of integral dose with higher-energy beams (4-6).

The use of integral dose in plan evaluation has been underutilized, although it could be a valuable metric, especially in cancer cases with curative intent for patients with long life expectancy. Therefore, it is somewhat surprising that so far integral dose (or total energy) minimization has been neglected in radiotherapy plan optimization. The aim of this work is to shed some light on the mathematical basis for the utilization of integral dose minimization in IMRT, and to present its application on a simple example.

\section{MATHEMATICAL FRAMEWORK OF INTEGRAL DOSE MINIMIZATION-BASED INVERSE IMRT OPTIMIZATION}

Energy minimization approaches are commonly encountered in physics where the solutions of many problems are very often based 
on this fundamental principle. The total energy imparted on an anatomical organ of interest is given by the integral dose and can be expressed according to Eq. 1,

$$
\begin{aligned}
E_{\text {total }} & =I=\sum_{i=1}^{N} d_{i} m_{i}=\sum_{i=1}^{N} d_{i} \rho_{i} v_{i} \\
& =\sum_{i=1}^{N} \frac{E_{i}}{\rho_{i} v_{i}} \rho_{i} v_{i}=\sum_{i=1}^{N} E_{i}
\end{aligned}
$$

where $d_{i}, m_{i}, \rho_{i}$, and $v_{i}$ are the dose, mass, density, and volume of dose voxel $i$, respectively. The summation is over all dose voxels contained in the volume of the organ, and $E_{i}$ is the energy imparted on voxel $i$. Mathematical incorporation of Eq. 1 in inverse optimization can be achieved after rewriting the representation as described in Eq. 2,

$$
F^{j}=\frac{1}{E_{\text {desired }}} \sum_{i \in V} d_{i} m_{i}
$$

where $F^{j}$ is the $j^{\text {th }}$ objective function, $E_{\text {desired }}$ is the desired integral dose, and the summation $i$ is over all voxels within the volume of a given anatomical structure. Note that for each anatomical structure, where integral dose minimization is required, only one objective can be specified, as opposed to dose-volume histogram (Dvh), or generalized equivalent uniform dose optimization (gEUD-based), where multiple objectives can be specified for the same anatomical structure (7-10). The minimization of course is applicable to organs at risk (OARs), where it is required that the delivered dose is as low as possible. The normalization to the desired integral dose $E_{\text {desired }}$ is performed such that a composite objective function (cf. Eq. 3) can be constructed, where individual objective functions $F^{j}$ can be expressed in terms of other dose representations $(7,11,12)$.

$$
F=\sum_{j=1}^{M} F^{j}
$$

Each individual term in the summation in Eq. 2 is always positive by construction since dose and mass can only be positive variables. Therefore, there is no need to introduce a quadratic form which requires minimization, as it is the case in Dvh- or gEUD-based optimizations $(7,12-14)$.

\section{EXAMPLE}

An example is presented to illustrate the basic points of the derived framework for energy minimization-based optimization, and to outline the differences with Dvh-based optimization. Figure 1 depicts a digital phantom in an axial view, on which the example will be illustrated. The phantom consists of three $10 \mathrm{~cm} \times 10 \mathrm{~cm} \times 10 \mathrm{~cm}$ cubical VOIs with densities of 0.2 (yellow), 0.8 (red), and 1.0 (green) $\mathrm{g} / \mathrm{cm}^{3}$. In the middle of the green VOI, there is a cylindrical target $3 \mathrm{~cm}$ in diameter and $3 \mathrm{~cm}$ in length. The high (red) and low (yellow) density regions are combined to form an "OAR" to which the dose is to be minimized through an inverse optimization. The target is irradiated with an anteriorposterior (AP) and a lateral (Lat) beam centered on the geometric center (isocenter) of the target. The two beams - AP and Lat are allowed to have only one IMRT segment each. Two IMRT plans
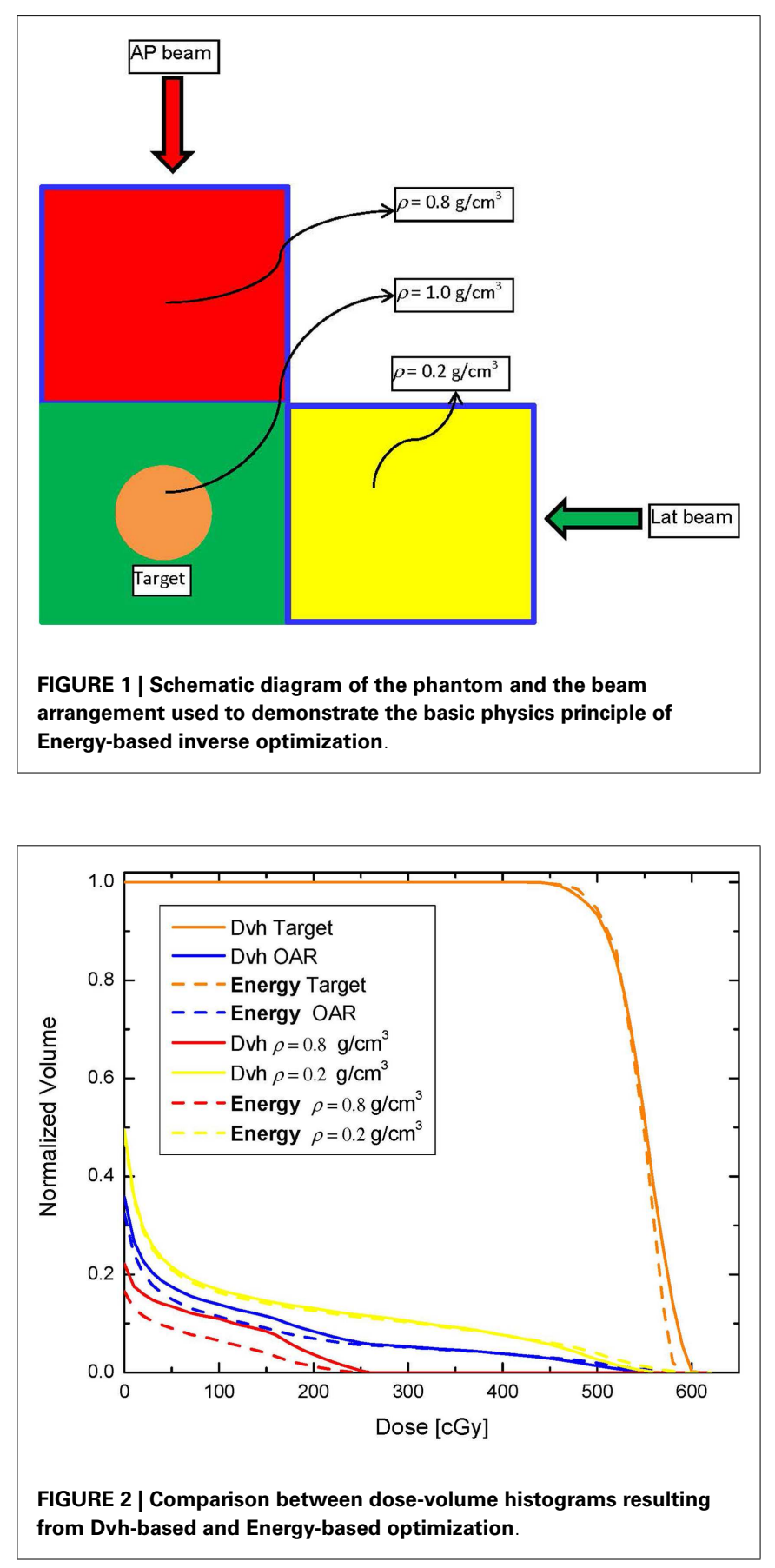

are generated with that beam configuration. In the first plan, the cost function for OAR dose optimization is constructed according to Eq. 4,

$$
F^{j}=\sum_{i \in V}\left(\frac{d_{i}-d^{j}}{d^{j}}\right)^{2} \Delta v_{i}
$$

where $V$ denotes the volume of the OAR for which $F^{j}$ is evaluated, $d_{i}$ is the dose in voxel ( $3 \mathrm{D}$ volume element) $i, d^{j}$ is the desired dose in each voxel, and $v_{i}$ is the normalized (with respect to the entire 
Table 1 | Integral doses and doses to $1 \%$ volumes of OAR, high density, and low density VOls.

OAR

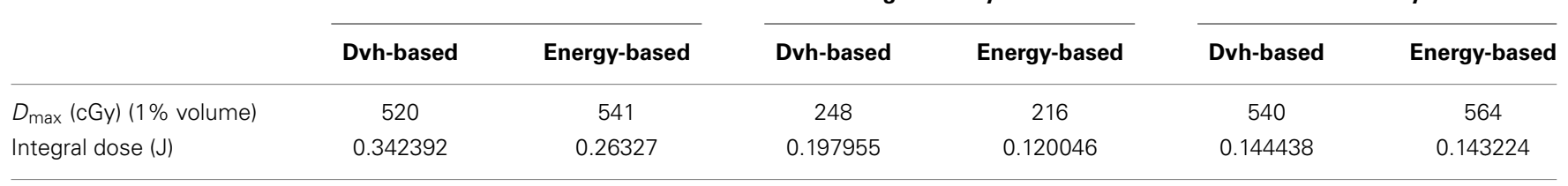

The doses to $1 \%$ volumes of the different regions are used as surrogates for maximum doses.

OAR volume) voxel volume (12). In the second plan, the OAR dose optimization is based on Eq. 2. Those two optimization schemes are termed Dvh-based and Energy-based, respectively. The inverse optimization was performed with a gradient decent method (7, $12,15-18)$ and was realized similarly to what other investigators have proposed (7). With each optimization the dose to the OAR is iteratively decreased until the standard deviation of the dose across the target reaches $6 \%$ of the prescription dose, i.e., no more than 30 cGy. The Dvhs of the two optimization approaches are presented on Figure 2. In addition, maximum and integral doses for the OAR, as well as the high and the low density VOIs are presented in Table 1. As can be noted on the figure and the table the high dose tails to the low density region (yellow) and the OAR (blue) are higher with Energy-based optimization, while the entire Dvh for the higher density region is lower than in the case of Dvhbased optimization. Therefore, in solving the global optimization problem, it seems that Energy-based optimization delivers more dose through the lower density region. Calculated doses to $1 \%$ of the OAR (as surrogate for maximum dose) are 541 and $520 \mathrm{cGy}$ with Energy- and Dvh-based optimizations, respectively. This difference indicates $\sim 4 \%$ difference in the maximum dose to the OAR. However, the average doses delivered to the OAR are 45 and $50.2 \mathrm{cGy}$, respectively, indicating that the Energy-based based optimization delivers $11.5 \%$ lower average dose to the OAR.

Furthermore, comparison of the integral doses to the irradiated volume, excluding the target, is evaluated. All three VOIs red, green, and yellow from Figure $\mathbf{1}$ are combined in a single structure and the integral dose to that volume is evaluated. The total imparted energy to that structure with Dvh-based optimization is $1.09977 \mathrm{~J}$, while with Energy-based optimization the imparted energy is $0.941815 \mathrm{~J}$. Therefore, in this very simple scenario the Energy-based inverse optimization results in an integral dose reduction to the entire volume in excess of $16 \%$. If only the integral doses to the OAR are considered (combination of red and yellow VOIs) then the total energy imparted to this region with Energy-based optimization is $\sim 30 \%$ lower, as can be concluded from Table 1.

\section{CONCLUSION}

A new approach based on energy-reduction inverse optimization was outlined. The mathematical framework for this optimization was defined. The energy-reduction approach was compared to the standard of care in inverse IMRT optimization, realized through Dvh-based optimization. Both optimization techniques were applied to a simple digital phantom for a very simple beam geometry, and their dosimetric properties were compared.
Energy-reduction approach resulted in lower average and integral doses to the volumes surrounding the irradiated target.

\section{ACKNOWLEDGMENT}

This work is supported by NIH grant R01 CA163370.

\section{REFERENCES}

1. Hall EJ, Wuu CS. Radiation-induced second cancers: the impact of 3D-CRT and IMRT. Int J Radiat Oncol Biol Phys (2003) 56:83-8. doi:10.1016/S0360-3016(03) 00073-7

2. Vanhavere F, Huyskens D, Struelens L. Peripheral neutron and gamma doses in radiotherapy with an $18 \mathrm{MV}$ linear accelerator. Radiat Prot Dosimetry (2004) 110:607-12. doi:10.1093/rpd/nch135

3. Podgorsak EB, Rawlinson JA, Johns HE. X-ray depth doses from linear accelerators in the energy range from 10 to $32 \mathrm{Mev}$. Am J Roentgenol Radium Ther Nucl Med (1975) 123:182-91. doi:10.2214/ajr.123.1.182

4. D'Souza WD, Rosen II. Nontumor integral dose variation in conventional radiotherapy treatment planning. Med Phys (2003) 30:2065-71. doi:10.1118/ 1.1591991

5. Pirzkall A, Carol MP, Pickett B, Xia P, Roach M III, Verhey LJ. The effect of beam energy and number of fields on photon-based IMRT for deep-seated targets. Int J Radiat Oncol Biol Phys (2002) 53:434-42. doi:10.1016/S0360-3016(02) 02750-5

6. Aoyama H, Westerly DC, Mackie TR, Olivera GH, Bentzen SM, Patel RR, et al. Integral radiation dose to normal structures with conformal external beam radiation. Int J Radiat Oncol Biol Phys (2006) 64:962-7. doi:10.1016/j.ijrobp.2005. 11.005

7. Wu Q, Mohan R. Algorithms and functionality of an intensity modulated radiotherapy optimization system. Med Phys (2000) 27:701-11. doi:10.1118/ 1.598932

8. Zhang X, Li X, Quan EM, Pan X, Li Y. A methodology for automatic intensitymodulated radiation treatment planning for lung cancer. Phys Med Biol (2011) 56:3873-93. doi:10.1088/0031-9155/56/13/009

9. Wu Q, Djajaputra D, Liu HH, Dong L, Mohan R, Wu Y. Dose sculpting with generalized equivalent uniform dose. Med Phys (2005) 32:1387-96. doi:10.1118/1.1997431

10. Bortfeld T. Optimized planning using physical objectives and constraints. Semin Radiat Oncol (1999) 9:20-34. doi:10.1016/S1053-4296(99)80052-6

11. Shipley WU, Tepper JE, Prout GR Jr, Verhey LJ, Mendiondo OA, Goitein M, et al. Proton radiation as boost therapy for localized prostatic carcinoma. JAMA (1979) 241:1912-5. doi:10.1001/jama.1979.03290440034024

12. Fredriksson A. Automated improvement of radiation therapy treatment plans by optimization under reference dose constraints. Phys Med Biol (2012) 57:7799-811. doi:10.1088/0031-9155/57/23/7799

13. Das SK. A role for biological optimization within the current treatment planning paradigm. Med Phys (2009) 36:4672-82. doi:10.1118/1.3220211

14. Widesott L, Strigari L, Pressello MC, Benassi M, Landoni V. Role of the parameters involved in the plan optimization based on the generalized equivalent uniform dose and radiobiological implications. Phys Med Biol (2008) 53:1665-75. doi:10.1088/0031-9155/53/6/011

15. Bortfeld T, Burkelbach J, Boesecke R, Schlegel W. Methods of image reconstruction from projections applied to conformation radiotherapy. Phys Med Biol (1990) 35:1423-34. doi:10.1088/0031-9155/35/10/007

16. Cho PS, Lee S, Marks RJ II, Oh S, Sutlief SG, Phillips MH. Optimization of intensity modulated beams with volume constraints using two methods: cost function 
minimization and projections onto convex sets. Med Phys (1998) 25:435-43. doi:10.1118/1.598218

17. Hristov DH, Fallone BG. An active set algorithm for treatment planning optimization. Med Phys (1997) 24:1455-64. doi:10.1118/1.598034

18. Hristov DH, Fallone BG. A continuous penalty function method for inverse treatment planning. Med Phys (1998) 25:208-23. doi:10.1118/1.598183

Conflict of Interest Statement: The author declares that the research was conducted in the absence of any commercial or financial relationships that could be construed as a potential conflict of interest.
Received: 21 April 2014; accepted: 27 June 2014; published online: 18 July 2014. Citation: Mihaylov IB (2014) Mathematical formulation of energy minimization based inverse optimization. Front. Oncol. 4:181. doi: 10.3389/fonc.2014.00181

This article was submitted to Radiation Oncology, a section of the journal Frontiers in Oncology.

Copyright (C) 2014 Mihaylov. This is an open-access article distributed under the terms of the Creative Commons Attribution License (CC BY). The use, distribution or reproduction in other forums is permitted, provided the original author(s) or licensor are credited and that the original publication in this journal is cited, in accordance with accepted academic practice. No use, distribution or reproduction is permitted which does not comply with these terms. 